\title{
A Robot System for High Quality Belt Grinding and Polishing Processes
}

\author{
Bernd Kuhlenkoetter \& Xiang Zhang
}

\section{Introduction}

Grinding and polishing are standard operations in the material processing. They are automated with the help of industrial robots in order to relieve human from laborious tasks and unpleasant environments and elevate the profitability of production nowadays, specially in the sanitary fitting industry. However, the systems known at present are adapted quite costly to other part geometries and operation cycles and are therefore economically applicable only for large batch sizes. Frequent changing of the robot program and system parameters also increase the cycle time and the cost of the whole manufacturing process. This problem will be more outstanding when the operating surface is a free-formed surface with very complicated geometrical shapes.

This paper describes a robot system which is specially developed for grinding and polishing free-formed surfaces with high quality and high efficiency due to the linkage of innovative robot technology, simulation technology and artificial intelligence methods. The robot system combines some different levels of automation, manual operation, partial automation and full automation. Highly labor-concentrated jobs will be done full automatically, for example detecting errors on the workpiece surface within the manufacturing process. Some modules are partial automated to help the operator plan the schedule and design the program. For operations that rarely occur a manual interference will be sufficient. A reasonable remaining of the manual work can keep the low costs of the robot system which is also acceptable for the enterprises. The robot system is flexible and suitable for the manufacturing of small and medium batch size, which is a difficult task for current robot-controlled grinding and polishing systems.

The automation in this robot system focuses on two parts. One is the quality assurance and the other is the process model that suggests the choice of the technical parameters for a high quality processing. To obtain a high quality surface, the robot system should have more accurate motion programs in the process optimizing. The new software system is developed exactly for this purpose. The software helps the operator design the grinding and polishing paths precisely and efficiently with CAD data of the grinding/polishing surface. In addition, the simulation of the grinding process will assist the operator to get more reasonable manufacturing paths and adopt the optimized parameters to the manufacturing process in order to obtain a high quality surface. The process model is specially for the grinding process that studies the relation between the various grinding parameters and the final amount of materials removal from the workpiece surface. The result of the process model makes the simulation of the grinding process possible. The 
operator can analyze qualitatively what the surface is like before the workpiece is really ground. This can direct the operator to modify the grinding path in advance and therefore reduce the rejection rate of the production processes.

In the next section, the grinding and polishing using industrial robots are briefly introduced. The system used and the key technologies in the system will be described in detail in section 3. In section 4 we give an account of our work to simulate the belt grinding process which is a very important part in the whole system. The sub-system of defect detection and classification is introduced in the section 5. We end with a summary and some main future plans of the system.

\section{Grinding and Polishing in the Manufacturing Chain}

It is the aim of grinding and polishing processing to improve the forming and dimensional accuracy as well as the surface finishing. Both processes play an important role as they are at the end of the product chain where processing errors lead to high rejection rates of the parts.

Specially in the sanitary fitting industry grinding and polishing are widely used to manufacture a free-form surface. Semi-finished workpieces are normally manufactured by casting. Through the subsequent grinding and polishing a high-quality shining surface is produced. In this case, the dimensional accuracy of the workpiece is only playing a secondary role. Fig. 1. illustrates five phases of producing a water tap fitting from the rough workpiece to the final product.
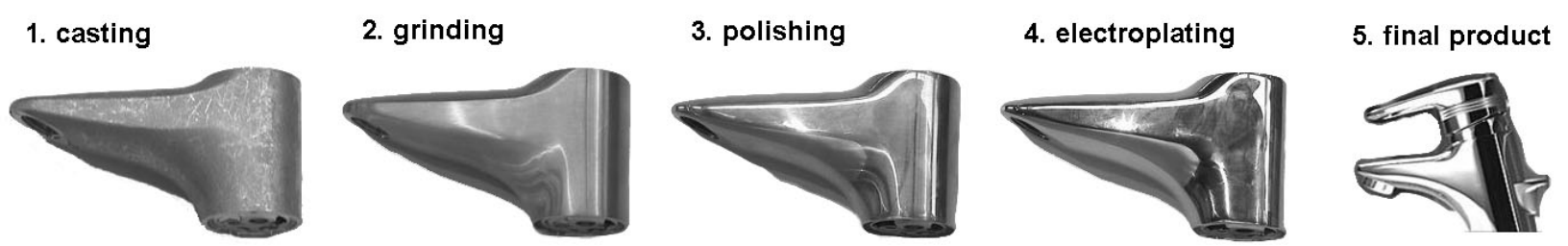

Figure 1. Producing steps in the manufacture of fittings electroplating, (5) end product

(1) casting, (2) grinding,

(3) polishing,

Traditionally grinding and polishing processes of such free-form surfaces are done by manpower. A worker holds the workpiece, feeds it towards the grinding/polishing machine and moves the workpiece along the paths that are decided subconsciously by experience, see left side of Fig. 2. In this manufacturing process, the worker has to suffer from the unpleasant environment, e.g. dirty air and loud noise. Additionally the job is hard and monotonous work so that the people cannot concentrate on it for very long time, so they cannot guarantee constant quality of the product. Nowadays, industrial robots are introduced into the manufacture of free-form surface grinding and polishing to minimize the costs while optimizing the quality. The robot, replacing manpower, holds the workpiece and moves along the paths that are predefined by technicians, see right side of the Fig. 2. To implement this process using industrial robots, the most important is to plan the grinding/polishing paths and generate the robot programs. Another practical problem to be handled is the occurring of errors in the previous casting phase. The casting process is characterized by high resulting dimensional and geometrical tolerances as well as quality fluctuations like blowholes and pores. These strongly variable starting conditions of grinding lead to unprofitable rejection rates and a very high manual testing procedures in the automated grinding and polishing processing. What is even more difficult for the 
realization of an automated solution is the fact that errors are only detectable after a part of the fine processing has been done and that the sensitive and strongly shining surfaces are difficult to measure.
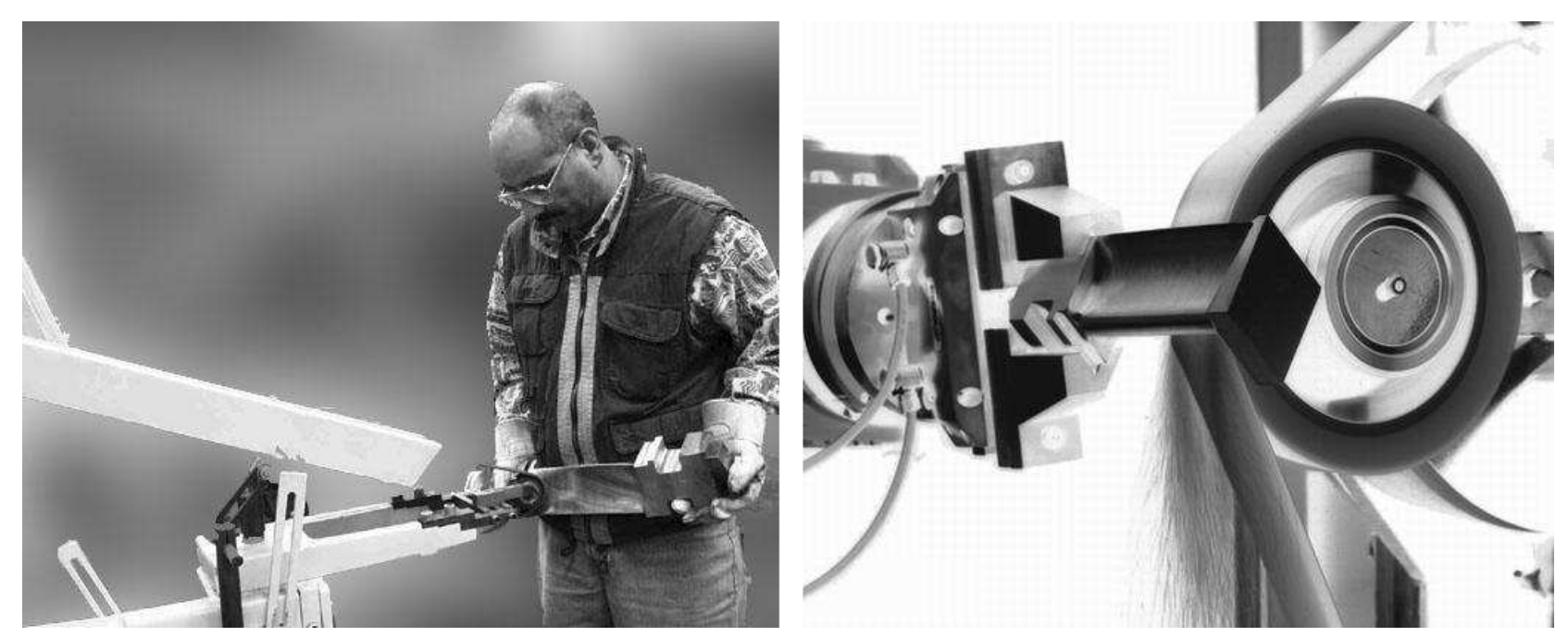

Figure 2. Manual grinding process and robot aided grinding process

The robot aided automation solutions known at present in the fields of grinding and polishing are especially and successfully used in the sanitary fitting industry. The profitability of these systems has been granted in the past years despite high national wage costs. With the advancing globalization of the markets, it is now faced with a high competition from low-wage countries where the grinding and polishing is done manually. Besides the grinding and polishing processes, the subsequent steps of manufacturing are also threatened to be shifted abroad. The cost of the total manufacture process should be reduced to face global competition. However, the high time and cost requirements for programming and optimizing have a negative effect on the profitability of industrial robot aided grinding and polishing cells (Schueppstuhl, 2003). Compared to conventional robot tasks these high requirements result from the clearly more complex, comprehensive and more accurate motion programs.

These requirements have of course an even more negative influence if new programming or adaptations become frequently necessary (Cabaravdic, 2003). The main reasons for this are an unfavourable ratio of the batch size to the variety of modifications and the occurring fluctuations in the process due to workpiece tolerances, and other errors in the upstream manufacturing process. The required level of automation of the procedures depends on the occurring frequency of the necessary optimizing work. For rarely occurring process malfunctions a manual interference is sufficient, while for more frequent occurrences a full automation must be realized. The techniques to be developed for a manual use differ considerably from a fully automated one. In manual procedures the focus lies on the efficient interaction with the operator whereas a full automation needs the development and integration of a complex measuring method, a data processing and process control.

\section{Innovative Robot System}

One aspect of the project aims at further developing the process specific offlineprogramming system, as approved in practice, in order to reach a higher efficiency than 
manual programming and optimizing procedure. The nowadays systems are designed for a universal use and similar to complex 3D CAD-systems what regards their layout and operation. Processes that do not need an extra path or parameter optimizing, such as palletizing, assembling or varnishing can be programmed efficiently with such software systems by high-qualified engineers and technicians in the planning department. However, there are no appropriate tools available for the grinding and polishing to optimize the phase directly at the robot cell.

The use of a conventional offline-programming system in grinding or polishing usually fails because of its complexity in connection with an unsuited qualification of the operators as well as lack of process specific functions. Therefore a high demand for according developments arose in this context. The intended system should adapted itself to small batch sizes and the case in which lots of modifications must often be carried out. The operator of the robot is in the centre of the decision-taking and should be given PCbased deciding guidance for the next step and according tools for an efficient program optimizing.

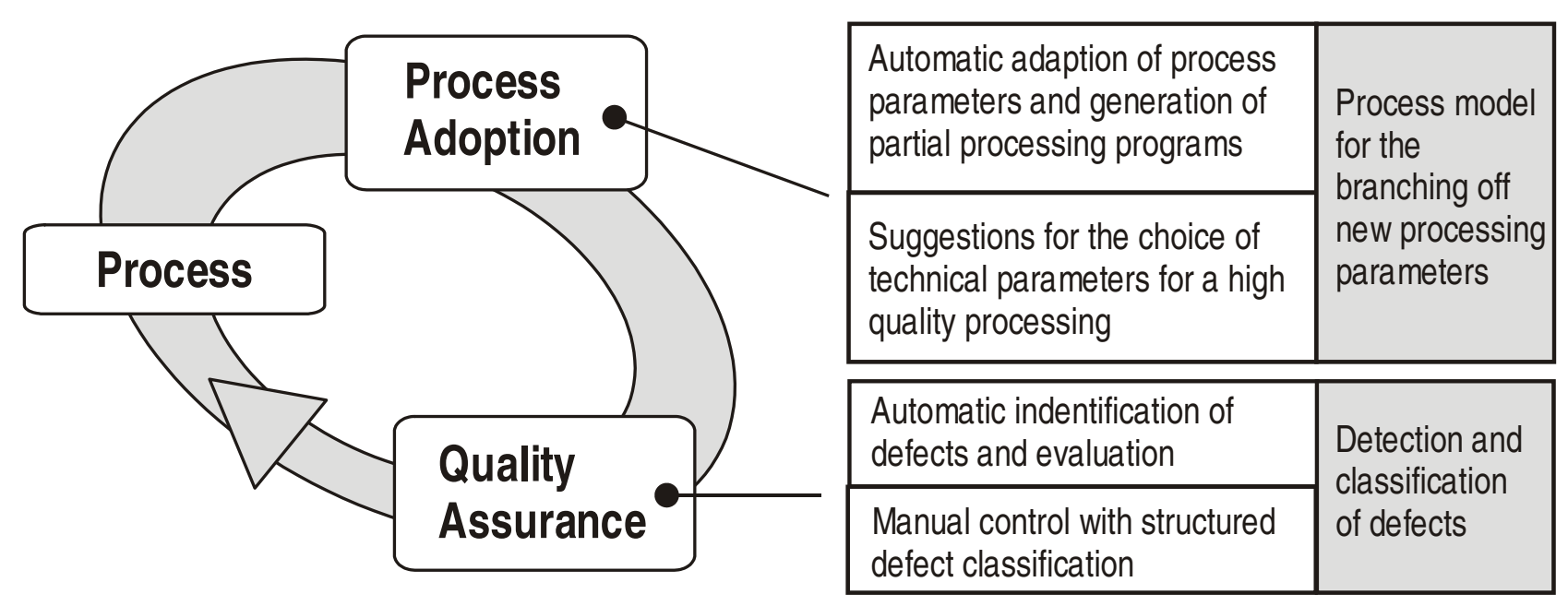

Figure 3. Central aspects of the system

Another aspect lies in the consideration of disturbing influences that frequently occur and which must be detected and compensated automatically. In practice, the most common disturbing influences are the existing defects on the semi-product from the casting phase or caused directly by the grinding processes.

Therefore, the error detection and classification as well as the deduction of parameter optimizing strategies through measuring methods and process control must be realized fully automatically, see Fig. 3.

A software system has been developed for a workshop programming of robot systems that provides an intuitively operable graphic 3D-user interface. The software provides process specific optimizing tools, specially for grinding and polishing. The software accepts the CAD model of the workpiece or its surface as input and helps the operator to generate the grinding or polishing path conveniently and practically. The software can generate the according robot programs for users.

With this software, the time required to design the paths is greatly reduced compared to the universal off-line programming system. It is very suitable for small or medium batch size manufacture and flexible to the requirements of frequent modifications of workpieces or paths. Fig. 4. shows the simple generating of grinding paths on the surface of the workpiece. Additionally, the software is extended by an adaptive consulting centre for the 
allocation of errors, causes of errors and compensation strategies and an internet connected process-know-how-database.

This software fills the gap between the multi-functional, however complex, offlineprogramming systems used in the planning department and the inefficient possibilities of the robot control used by the operator for the program optimizing.

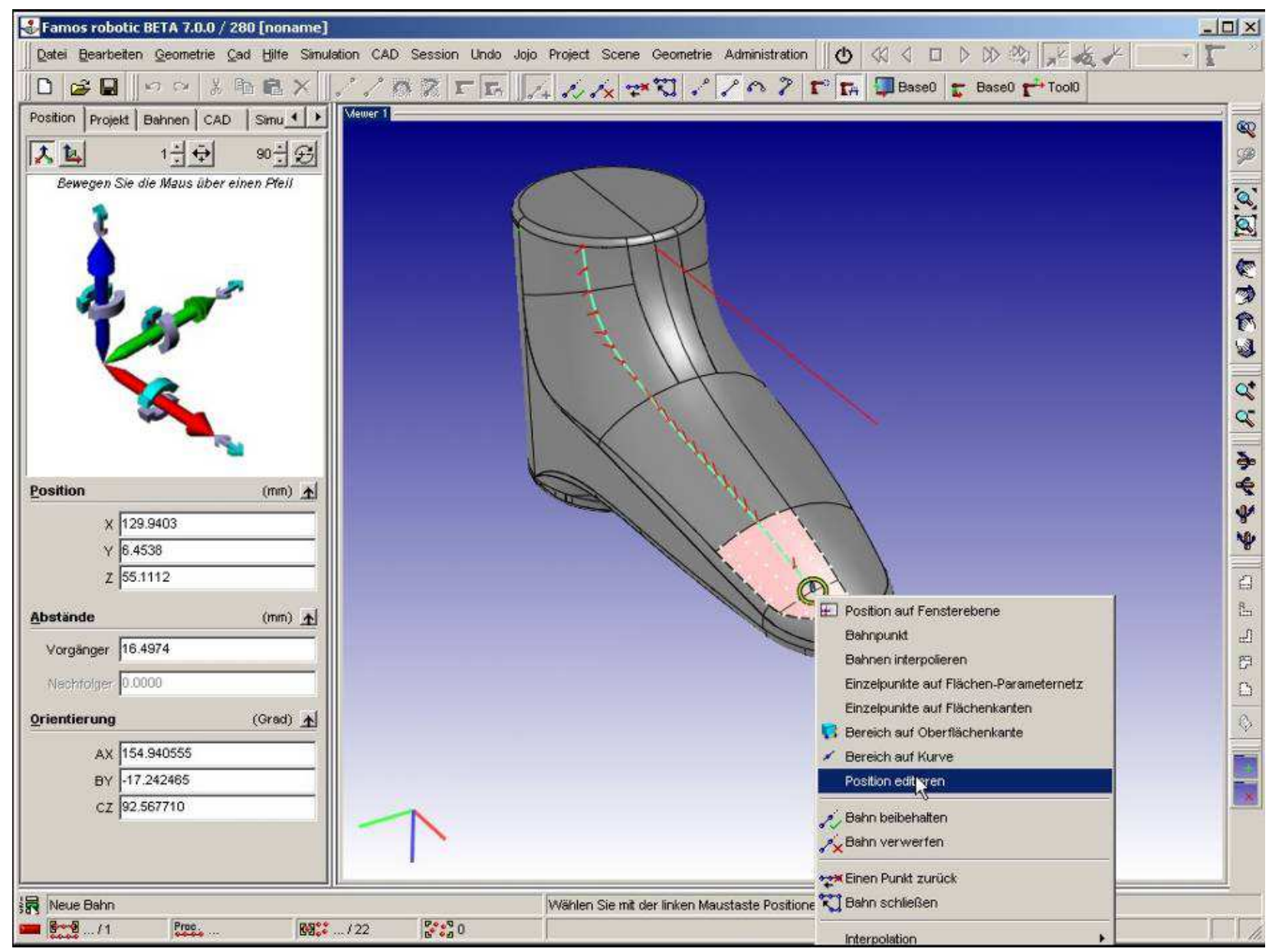

Figure 4. User-orientated offline-programming and simulating system (Carat Robotic Innovation, 2004)

A higher tolerance towards changing starting qualities of the workpiece is achieved by combining image processing/measuring systems, grinding and polishing process models, and adaptive control technique and intelligent software components. The "see and evaluate" of processing errors on high shining surfaces is even difficult for the untrained human eye.

Furthermore, errors in the workpiece material in the process chain of rough grinding, finish grinding and polishing can be often detected only after a part of the processing has been done, or at the end.

Therefore, another requirement has arisen for developing a fully automatic working process chain for the industrial robot-aided grinding and polishing based on the measurements of an image processing system so that an optimum surface quality is obtained despite fluctuating starting conditions. Fig. 5. below illustrates the working flow of the automatic detection and classification system. The central hardware is a closed vision system that can provide suitable illumination and take photos of the workpiece surface. The software of the system is responsible for finding the defect position and classifying the defects by analysing the grayscale photos taken by the vision system. 


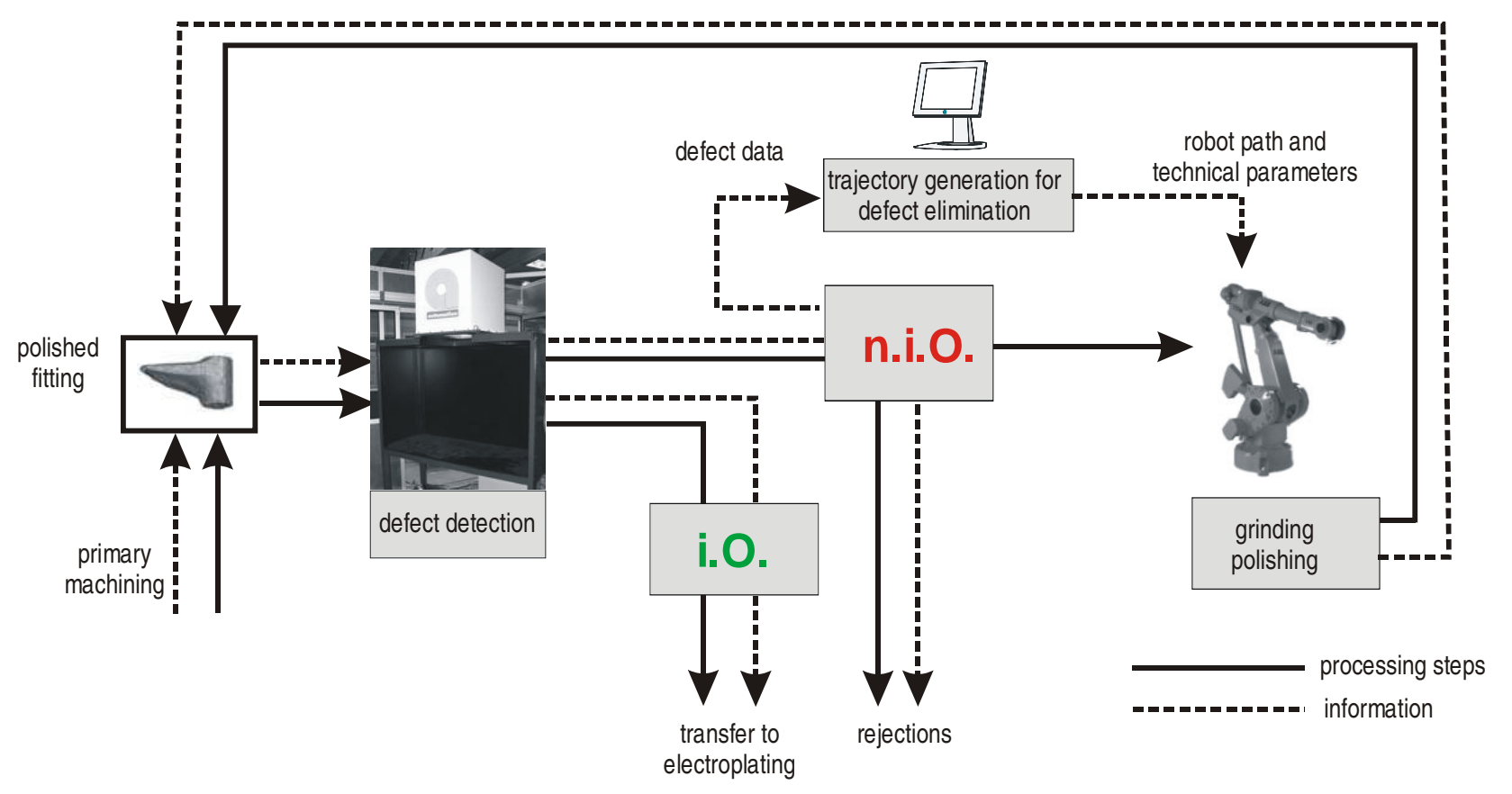

Figure 5. Flow chart of the fully automated process chain

After polishing, the workpiece is transferred to the vision system to evaluate. The workpiece will go to the next manufacturing step when no defects are found on the surface.

A workpiece that has remediless defects will be rejected or discarded while the rest is ground and polished according to the individual defect data. The retouched workpieces are fed into the automatic vision system again until they are either accepted as qualified or discarded as defectives. The compensation machining process for detected errors on the surface is already realized in the system.

\section{Simulation of Belt Grinding Processes}

The operator can design the grinding and polishing path easily by using the software as shown in Fig. 4. However, it is a highly experience-related job to decide the paths on the workpiece surface. A skilled operator generates the paths and according programs that result in a good surface quality and low rejection rate while an untrained operator reaches a high rejection rate that damages the profitability of the robot system. The individual operator produces inconsistent standard of design and causing a various surface quality of the workpieces. Therefore, the belt grinding process and robot system should be studied in detail with a process simulation being necessary at the same time. Both path planning and parameter selection of the robot system will benefit from the results of the simulation afterwards.

\subsection{Introduction of Belt Grinding Processes}

Belt grinding is a machining process with a geometric indeterminate cutting edge. The grinding belt (cutting tool) consists of coated abrasives and is attached around at least two rotating wheels.

The part to be ground is pressed onto one of these wheels, which is the contact wheel. Fig. 6. shows the belt grinding process. The material is cut off under non-permanent touch between workpiece and abrasives. 


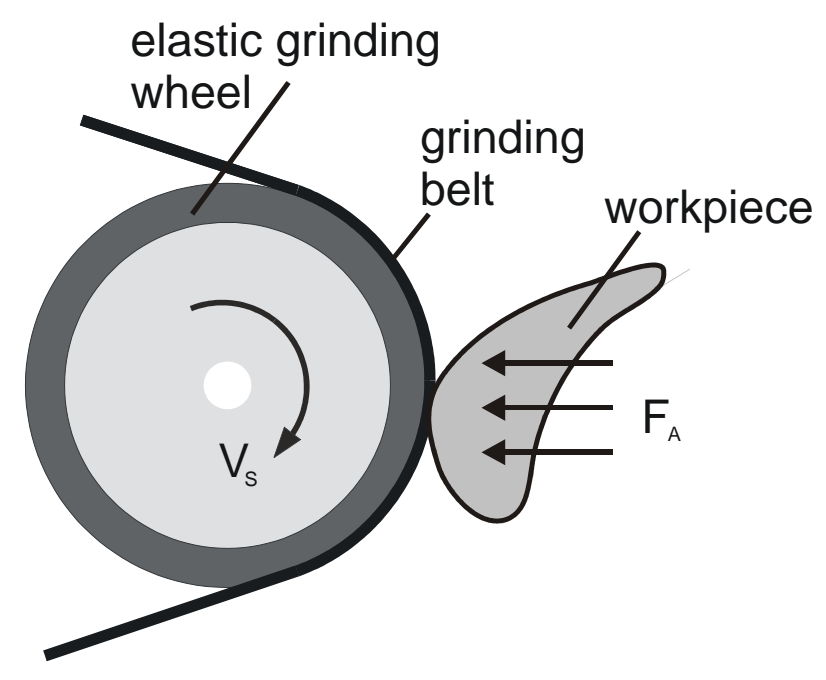

Figure 6. Belt grinding process with elastic wheel

The variant of the belt grinding with elastically deformable contact wheel is especially suitable for the finishing of free-form surfaces because the elastic contact wheels allow a flexible processing through their adaptation to the part surface. Compared to other finishing processes, belt grinding with elastic contact wheel is characterized by a higher removal rate with comparable surface quality. The most important advantages of this process variant are:

- The elasticity of the contact wheel leads to a better form adaptation to the surface of the workpiece compared to a rigid contact wheel and therefore produces a higher quality of sculptured surfaces.

- The compensation of the infeed and orientation error of workpieces or tools is possible to some degree.

- During a long phase the grinding belt wear factor is approximately constant. Thus the belt wear does not affect the machining process in this phase.

\subsection{Removals in Belt Grinding Processes}

The most important point in simulation is to calculate the removals from the workpiece surface at a discrete point of time. The simulation of the grinding process is more difficult than those precise operating processes, e.g. milling, because the removal of the surface cannot be obtained directly by calculating the swept volume through CAD data and tool shape. The cutting tool in belt grinding is the grinding belt that is coated by hard abrasives. In machining, the grinding wheel rotates and the belt rubs and strikes the workpiece surface.

Because shape and distribution of the abrasives on the belt are non-uniform and rather disordered, the belt grinding processes are considered as a cutting processes with an indeterminate cutting edge. Another problem is the elasticity of the tool (grinding wheel) that can cause a strong force variation between the grinding wheel and the workpiece. Calculating removals is therefore not a purely geometric computation, but a quite experience-based process. Besides the material of grinding belt, quite several other parameters can simultaneously work on the final removal from the workpiece surface, for example elasticity of grinding wheel, temperature and so on. The removal from the workpiece surface is a function of a group of factors.

To model this, Hammann presented a linear experiential formula (Hammann, 1998; Schueppstuhl, 2003): 


$$
r=K_{A} \cdot k_{t} \cdot \frac{V_{b}}{V_{w} \cdot l_{w}} \cdot F_{A}
$$

where $\gamma$ is the removal; $K_{A}$, a combination constant of some static parameters; $k_{t}$, the grinding belt wear factor; $V_{b}$, the grinding velocity; $V_{w}$, the workpiece moving velocity; $l_{w}$, the length of the grinding area; $F_{A}$, the acting force. Using this model, one can do many experiments making only one factor variable and all other factors unchanged at the same time. Then, the experimental results can be combined to determine the coefficients in the model. The parameters in the model are all one-valued which means all inputs of the model are indicated by only one value, for example the acting force $F_{A}$ is the global force between the workpiece and grinding wheel. The global grinding model is sufficient to the operating of simple shapes. But it is obvious that they are incapable to free-form surface grinding because not only the total removal but the local removals distribution (removals at small sub-area) are neccessary to be known.

Removals distribution results from the contact force distribution in the grinding process. The detailed local force distribution information (not only the global force FA) should be obtained before the removals distributions are considered. Normally, the Finite Element Method (FEM) is the traditional way to calculate the contact forces according to the initial contact situation. The FEM deals with this contact problem as an ideal Signorini contact problem (Krause, 2001) which solves an optimization problem by a contact energy minimization principle. Considering the process locally, the surface of the treated workpiece is split into a number of "finite elements" (Hammann, 1998; Schueppstuhl, 2003), assuming constant distribution of the contact pressure and cutting speed at each element. Hence, the contact pressure must be calculated for each element. The overall contact pressure distribution for one contact situation is then given by all local element pressures. Local removals can be calculated through a multi-factor statistical analysis in the second modeling step. Fig. 7. illustrates the framework of the process model for freeform surface grinding. First of all, the geometry and elasticity information are constructed as the initial contact situation which is the input of the FEM model. Then, FEM works out the distribution of force between the workpiece and the grinding wheel. Finally, the distribution of force together with other process parameters are given into the local multifactor analysis model to calculate removals distribution.

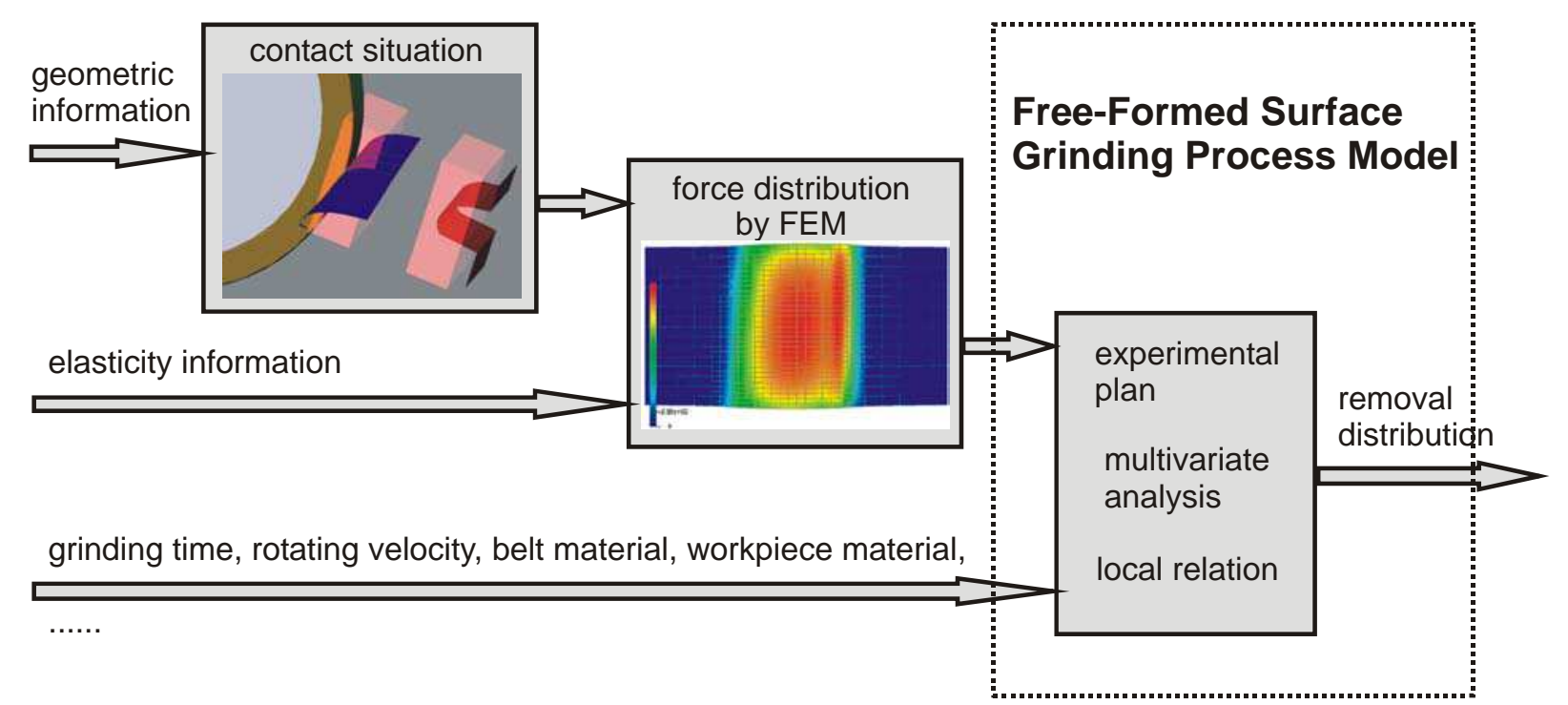

Figure 7. Process model for free-formed surface 


\subsection{Simulation of Robot Controlled Belt Grinding Processes}

Fig. 8. is a flow chart of simulation processes (Schueppstuhl, 2003; Kreis\& Kneupner, 2001a, 2001b). The planned path is known at first and the grinding process is divided into some discrete time intervals. At any time point, the initial contact situation can be reached by the current CAD model and the path. The next step is to calculate the force distribution and then get the removals distribution by the process model. The current CAD model is updated and moves to the next time interval until the path end. In this simulation framework there are four important parts:

- Initial contact situation modeling

- Force distribution calculating

- Process model

- Workpiece model

Additionally, a direct control system should be implemented if an on-line control of the robot is required (Kneupner, 2002; 2004).

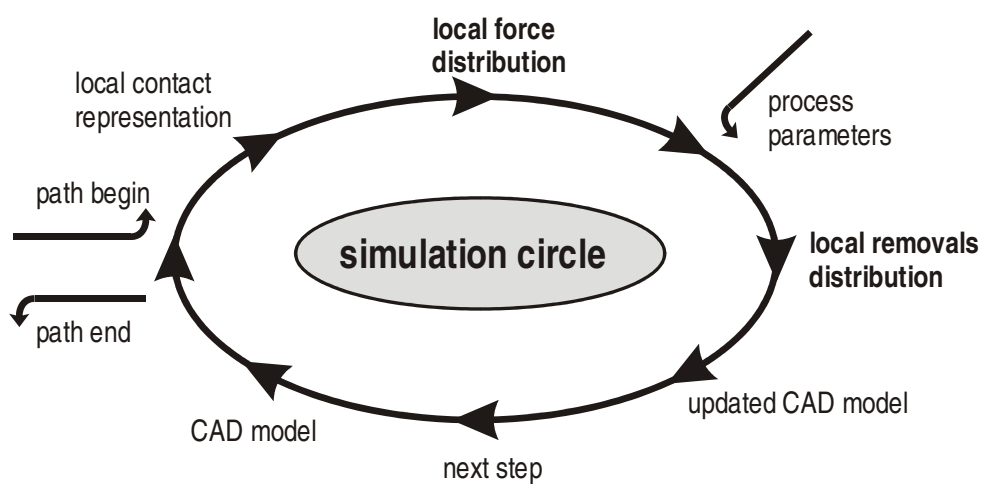

Figure 8. Simulation circulation

While simulating, the removal is calculated by the process model. Especially for surfaces with a small radius, the change of the surface can be dramatic. Therefore it is not possible to calculate a complex geometry using a so-called swept volume which interpolates the removal between two different removal calculations. Instead, the shape of the workpiece is changed directly after one removal calculation and the next calculation is based on the new geometry. An important effect which should be taken into account in simulation processes is the so-called "cut loose" effect (Kneupner, 2004). It means that the tool will be not in contact with workpiece any more after a specific time of grinding if the workpiece is not moved because all material in between will be worn out. While this happens, the actual removal rate will slow down. It is clear that such a process can only be simulated if the workpiece model is updated after a removal calculation. Thus, the time interval should be small enough to neglect the decreasing removal rate because of the "cut loose" effect. Moreover, a fast calculation is essential for practical reasons. The cycle time is driven by an external cycle time. As it is possible to calculate the position of the tool in relation to the workpiece with a high accuracy within the robot interpolation cycle time, we use a multiple interpolation time cycle. This is an amount of time small enough for neglecting the shape-change within a calculation. The Height Model is put forward in our project to describe the initial contact situation specially considering the characteristics of belt grinding process, assuming that workpieces for grinding are idealistically hard and stiff without deformation and that the grinding wheel is made of soft material with a known 
elasticity, which at the same time is also a prerequisite of the Signorini contact problem. So when the grinding wheel contacts the workpiece, it deforms according to the geometry of the workpiece surface and actual infeed. To describe such a situation, Height Model divides the contact area into a mesh where then the initial contact situation of grinding wheel can be encoded as a group of so-called Heights, which are intervals between the base plane and deformed surface of the grinding wheel at all mesh points (see the right part of Fig. 9.). The base plane is always vertical to the infeed direction and has invariable distance to the wheel center. The normal vectors of all contact points on the deformed surface are also recorded for later use.
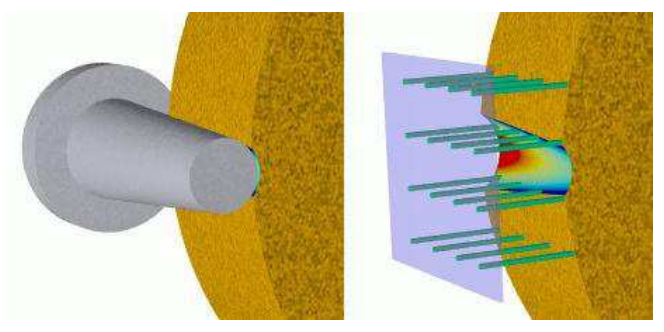

Figure 9. Height Model

The Height Model is actually a discrete description of an initial contact situation in which mesh size is a control factor for different precision requirement. In this way, each contact situation can be described by a heights matrix $\mathrm{H}$, which has the following form:

$$
\mathbf{H}=\left(\begin{array}{cccc}
h_{11} & h_{12} & \ldots & h_{1 n} \\
h_{21} & h_{22} & \ldots & h_{2 n} \\
\vdots & \vdots & \ddots & \vdots \\
h_{m 1} & h_{m 2} & \ldots & h_{m n}
\end{array}\right)
$$

where $\mathrm{m}, \mathrm{n}$ is the mesh size in two directions.

The contact situation between workpiece and tool can change very fast during the processing of sculptured surfaces, causing a strong removal variation. This fact asks for quick calculation of force distribution in real time. The traditional way to calculate the force distribution is resorting to the FEM that consider the contact problem as an ideal Signorini problem. Blum and Suttmeier (Blum et al. 2000; 2003; Suttmeier, 2001) worked out a FEM model. The FEM model has to achieve the optimization solution through iterating steps each time when a new contact situation is presented. Thus, it is very timeconsuming. Although the model used an optimized mesh discretization, it still requires about 15 minutes for calculating one contact situation. This is far away from the demand for real time simulation. The force distribution calculation is the practical bottleneck of the real-time simulation. To meet this, a new force distribution calculation model (Zhang et al., $2005 a ; 2005 b)$ is worked out to accelerate the calculation. The idea is that the neural network or Support Vector Machine serves as an approximation of the FEM model to learn the non-linear mapping from the initial contact situation to force distribution. The expensive iteration process is shifted in the training process of the model, but not in run time resulting in a dramatic reduction of the calculating time which makes the real time simulation of the grinding processes possible. 
After the local forces are known, it is time to decide the parameters in the process model. The experiments and previous research (Koenig, 1996; Meyerhoff, 1998; Hammann, 1998; Schueppstuhl, 2003) showed that an overall mathematical description of the belt grinding process is not possible because a complete list of the influential factors cannot be determined exactly.

In the process model, only eight parameters are selected out which are:

1. force floc $(\mathrm{N} / \mathrm{mm} 2)$

2. rotating rate of grinding belt $\mathrm{vb}(\mathrm{m} / \mathrm{s})$

3. grinding time ts (s)

4. local radius of the workpiece rloc $(\mathrm{mm})$

5. the grain size of grinding belt $\mathrm{kb}$

6. belt tension $\mathrm{fb}(\mathrm{N} / \mathrm{mm} 2)$

7. contact length lc ( $\mathrm{mm})$

8. material of the workpiece mw

The influential factors of belt grinding can be determined only statistically due to the geometrically indeterminate cutting edge. The parameter values must be in a reasonable range in order to predict the removal precisely enough.

Statistical design of experiments is applied for the modeling of local relations. An essential aspect of the statistical design of experiments is the fact that several factors are varied simultaneously from a single experiment to the next one. In order to implement that, a experimental plan is used by the statistical design of experiment. With the help of such an experimental plan, more complex relations can be modeled. In our case a full factorial experimental plan with $2^{8}=256$ single experiments is used for all the 8 influential factors on two factor levels.

The local removals as the response variables are measured after every individual experiment using a sensor device. The experiment data are listed in the Table 1.

The influence of wear is minimized by the use of wear resistant grinding belts. A heat influence can be minimized through a suitable selection of grinding times. The grinding time for every individual experiment must not be too long so that the grinding belt is not heated up. This experimental plan has been done only partially and is still in work.

\begin{tabular}{|c|c|c|c|c|c|c|c|c|}
\hline \hline & $\begin{array}{c}f_{l o c} \\
(\mathrm{~N} / \mathrm{mm})\end{array}$ & $\begin{array}{c}v_{b} \\
(\mathrm{~m} / \mathrm{s})\end{array}$ & $\begin{array}{c}t_{s} \\
(\mathrm{~s})\end{array}$ & $\begin{array}{c}r_{\text {loc }} \\
(\mathrm{mm})\end{array}$ & $k_{b}$ & $\begin{array}{c}f_{b} \\
(\mathrm{~N} / \mathrm{mm})^{2}\end{array}$ & $\begin{array}{c}l_{c} \\
(\mathrm{~mm})\end{array}$ & $m_{w}$ \\
\hline 1 & 0.01 & 10 & 5 & 30 & $\mathrm{P} 60$ & 0.1 & 5 & 1.0037 \\
\hline 2 & 0.5 & 30 & 15 & 200 & $\mathrm{P} 120$ & 0.4 & 50 & brass \\
\hline
\end{tabular}

Table 1. The technical data for all parameters

\subsection{Simulation Examples}

Four parameters are used to simplify the definition of the workpiece surface, the infeed $x$, the local curvature (the reciprocal of the radius) in $\mathrm{y}$ and $\mathrm{z}$ direction and the turning angle. The simulation results can imply how this local parameters act on the local removals. Fig. 10(a). illustrates the simulation result with different infeeds. The length of the pillar on the workpiece indicates the grinding infeed and colors on the workpiece surface denote the local removal distribution. The infeed starts with zero at the beginning of the path and reaches the maximum at the end of the path. The removal is growing when the infeed increases. This is because a bigger infeed leads to a larger deformation of the elastic grinding wheel and bigger acting forces. Fig. 10(b). illustrates the simulation result with 
different local curvatures in y direction. The length of the pillar on the workpiece indicates the local radius of the workpiece surface. The green pillar means the local surface is concave and the red pillar means the local surface is convex. The infeeds are the same along the grinding path. It shows that the convex part has a bigger removal than that of the concave part which conforms to the practical experiments. Fig. 10(c). is the result with different local curvatures in $\mathrm{z}$ direction. Fig. 10(d). is the simulation result of grinding along a simple equal-infeed path. The workpiece has a small turning angle that changes the local contact situation between the workpiece and grinding wheel, so the removals are also not distributed uniformly.

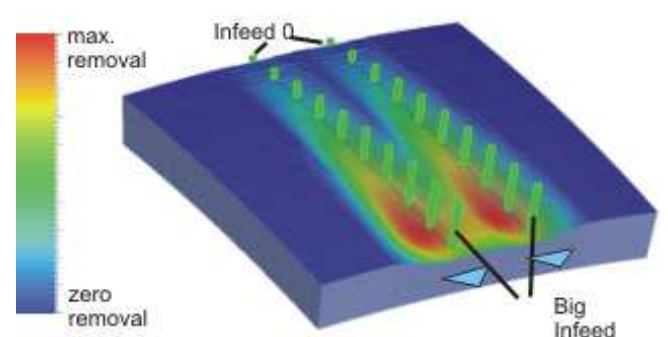

(a)

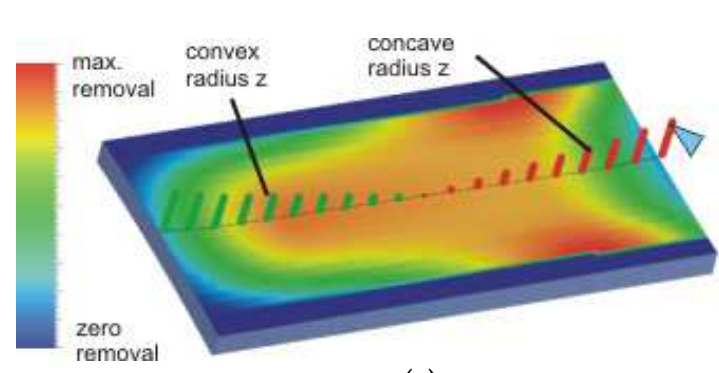

(c)

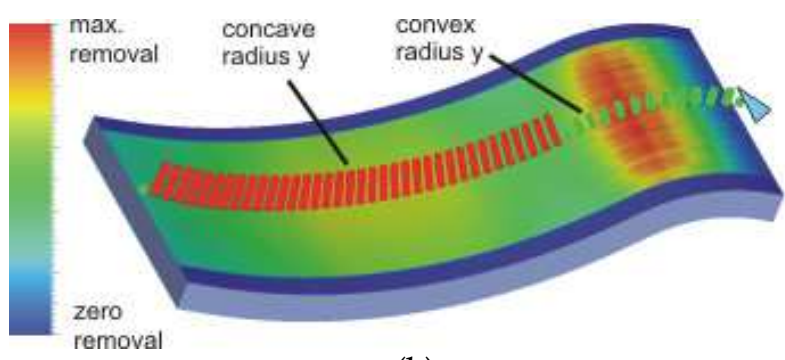

(b)

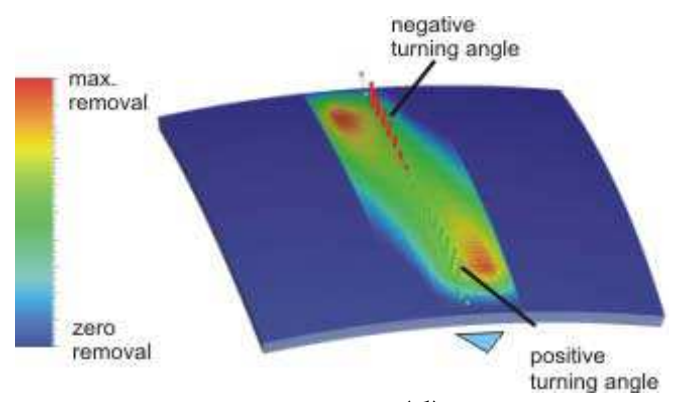

(d)

Figure 10. Simulation results (a) with different infeeds, (b) with different longitude local radii, (c) with different latitude local radius, (d) with turning angle (Schueppstuhl, 2003)

\section{Defects Detection and Classification on Workpiece Surface}

As mentioned in the section 3, another troublesome work is to find and identify the defects on the surfaces that are caused in the previous casting phase. In the past, this job was done by well-trained workers. They checked the surfaces for potential defects and classified them into pre-defined categories. In order to reduce the wage cost, this process is also attempted to be replaced by an automatic defect detection and classification with a vision system.

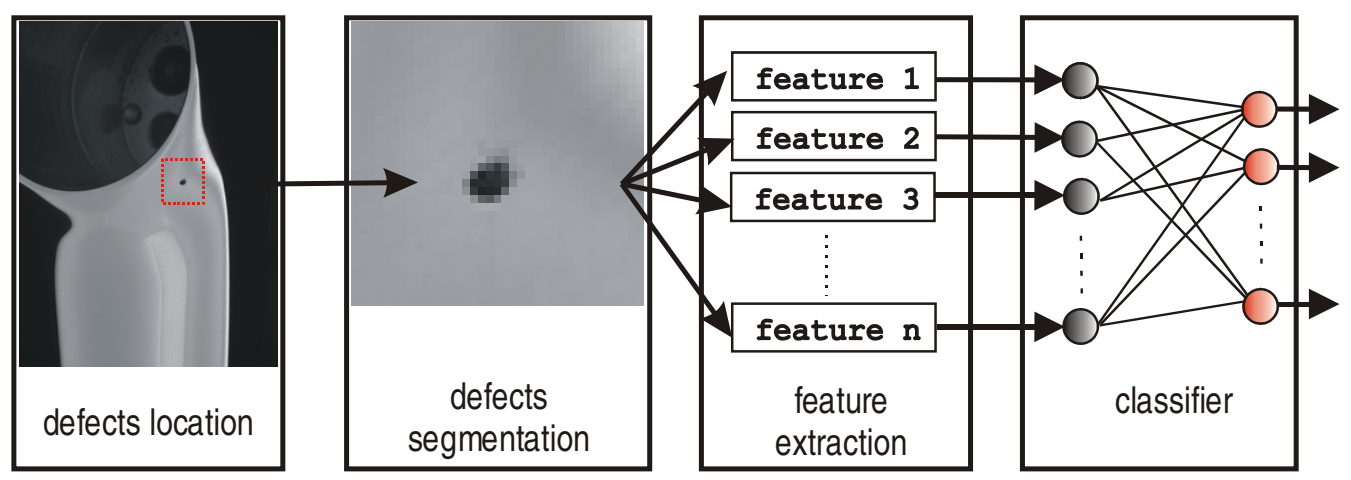

Figure 11. The framework of the automatic identification and classification system 
The planned classification system mainly consists of four parts: defects location, defects segmentation, feature extraction and classification. The four parts in the system act sequentially. Fig. 11. shows the framework of the classification system.

At the beginning, the system detects and locates the defect in the grayscale bitmap obtained from the vision system. Before encoding the bitmap into some meaningful feature values, the area of the defect in the image should be pre-processed and segmented. In this phase the separate defect image is obtained and is ready for feature extraction. The feature extraction is the most important part in the system. It defines the rules of describing and expressing the defects inside an image in a form that a classifier can understand. At first, those features should be found which are useful to differentiate one defect from another,.

The features are not limited to the shape feature, but can be the texture features and some statistical features of the segmented image. Then, an effective way should be found to encode such selected features, specially in a mathematical way. After the data preprocessing, the feature data are applied as the training data to the classifier. Many artificial intelligent techniques have the capability of multi-class classification, e.g. k-NN, MLP network, RBF network, SVM, etc.

The vision system adopt a specific illumination method to get a light color surface lying on the dark background. A small window is then applied on the grayscale picture of the surface. By analyzing the grayscale distribution in each small window, the defects can be located precisely because the defects are of deep color and surrounded by the light color surface. Fig. 12. shows 5 different defects selected from the total 19 classes that have been found on the workpiece surface.

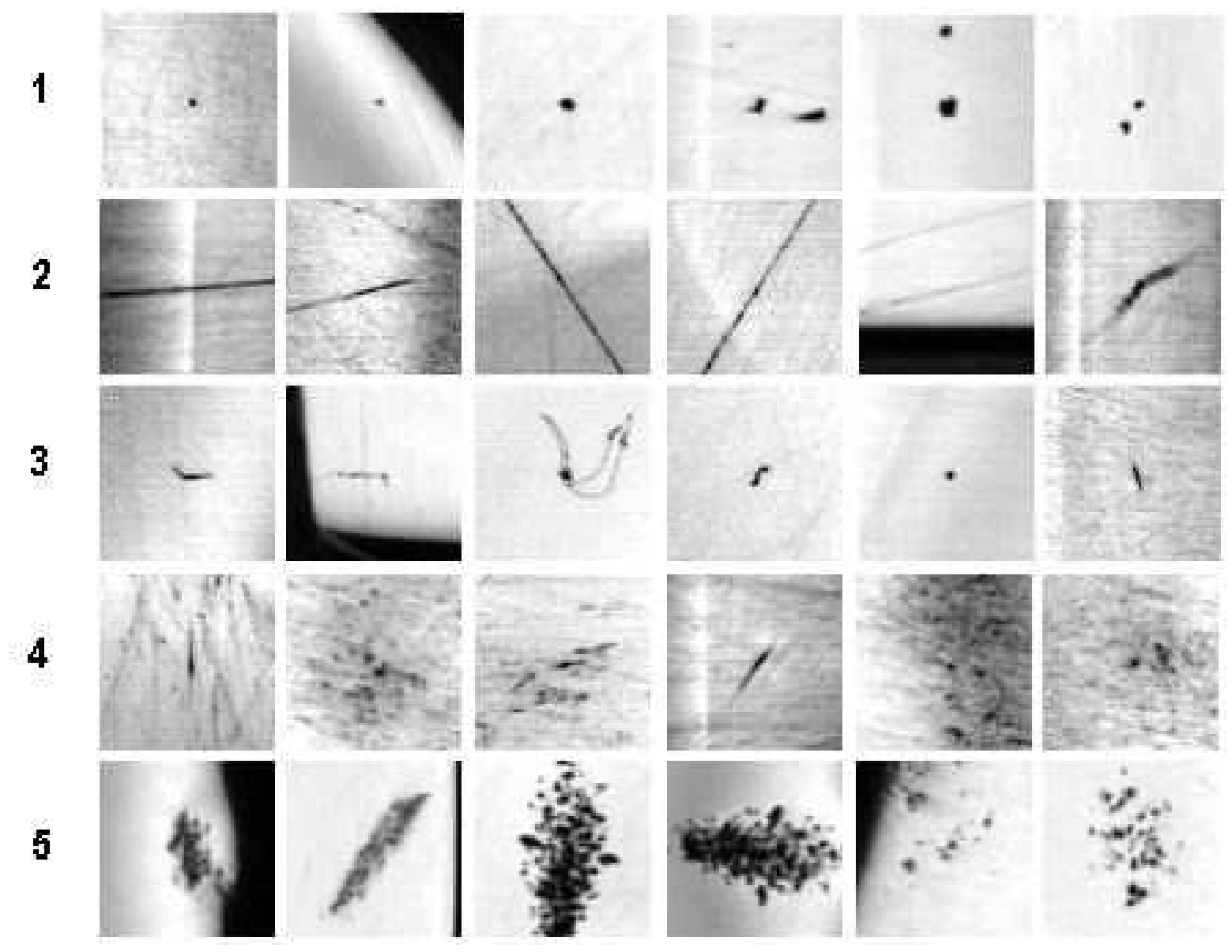

Figure 12. Example of 5 classes of defects. (1)pore, (2)subtle line, (3)fluff, (4)polishing shade (5) casting peel 
Technicians differentiate the defects by experience. Actually such experience involves some rules. For example, a small black solid circle in the white background may be the "pore" defect; a long stroke on the surface may be a "subtle line" defect. In other words, a technician draws a conclusion by combining some attributes of the defect in the segmented image, e.g. size, grey scale distribution and so on. All these attributes used by technicians (no matter intentionally or subconsciously) to discriminate defects are called valid features of the defect.

The length and width of the defects are the simplest valid features. It is very easy to see that such size information are the reason for a technician to differentiate the defects. However, not all features used by human beings are as straightforward as the shape features such as length and width. The fact is that you use some features to classify but you do not know you use them as a base. From the result of some other similar projects (Lampinen\&Smolander 1994; Iivarinen\&Visa 1998), three kinds of features are normally extracted to identify and classify the object, shape, texture and some statistical features of the image, e.g. grayscale histogram. It is not obligatory to include all those features as the input to the classification process. It depends on the project itself to decide which features to include.

Two basic principles should be hold in mind:

1. The features extracted are sufficient to separate different defects.

2. The features that is not helpful to discriminate the defects are not extracted to the classifier.

The first principle is a precondition of the system to function. Different defects are represented by features of different values. Think of the classifying of an apple and a pear, for example. Only by the shape feature it is not possible for people to tell whether it is an apple or a pear. It does not comply with the first principle because the apple may have the same and resemble the outer shape of the pear. The second principle is to ensure the generalization of the classifier.

Those features that are not helpful to distinguish the objects are not selected best. For example, the weight is not a feature that should be used because an apple is possibly heavier or lighter than a pear. Unnecessary features act like noise in the system. They distract the classifier from the right way and influence the performance of the classifier. The quantitative measurements of features are called descriptors. The way to measure shape are called shape descriptors while texture features are expressed by texture descriptors. There are many different descriptors available. Descriptors design and selection is an important research topic in the classification system. The current system uses only some simple shape descriptors, e.g. length, breadth, compactness and roundness of the defect. The right classification ratio is only about $30 \%$ now. There are three reasons for the low classification ratio.

The first one is the lack of the effective descriptors to account for the defects. The second reason is that the system did not use the up-to-date intelligent classifier and the last one is that there are two many pre-defined classes of defects (19 classes). Some kinds of defects are so similar that even skilled people can not differentiate them from each other. For example, "polishing shade" is similar to the "subtle line". When only analyzing the grayscale pictures, the "pore" defect is difficult to separate from the "fluff" defect by the operator in some cases, even though they are different in reality, see Fig. 12. The classification of defects is still under development. It is supposed to be able to reach an $80 \%$ right classification ratio by well-presented feature extractors and tailored classifier in the future. 


\section{Summary}

Due to the tendency of the globalization of markets and competition from low wage countries, it is profitable for the industry to further automate the robot-controlled grinding and polishing system, especially in the sanitary fitting industry. The high automation level of the robot system not only relieves the human being from laborious tasks, but also elevates the efficiency of the whole manufacturing process by producing a steady product surface quality. A framework of such a robot system is put forward in this paper. The automation of the robot system focuses on two parts. One is to facilitate the grinding and polishing paths planning processes. Software is developed to help the operator to generate the grinding and polishing paths quickly and easily. It offsets the incapability of universal off-line programming systems for grinding and polishing. With this new software the tasks that had to be done by high-specialized staff before can now be taken over by normal operators. To help the operator to design the paths, the belt grinding process and its simulation are carefully studied. Another key point of the automation is to automatically find and classify the defects on the workpiece surface into some pre-defined classes in order to apply an according compensating machining process. The detection of the defects is already realized while the classification should be improved in the future work.

This research and development project was funded by the German Federal Ministry for Education and Research (BMBF) within the national "Research In Production For Tomorrow" Programme and managed by the Project Agency for Production and Manufacturing Technologies (PFT), Forschungszentrum Karlsruhe

\section{References}

Blum, H; Suttmeier, F.T. (2000) An adaptive finite element discretisation for a simplified signorini problem. Calcolo, 37(2):65-77.

Blum, H; Schroeder, A; Suttmeier, F.T(2003). A Posterori Error Bounds for Finite Element Schemes for a Model Friction Problem. Conference Proceedings of Simuation Aided Offline Process Design and Optimization in Manufacturing Sculptured Surface, pp 39-47, Witten-Bommerholz, Germany.

Cabaravdic, M; Kneupner, K; Kuhlenkoetter, B. (2003) Methods for efficient optimisation of robot supported grinding and polishing processes. International Conference on "Trends in the Development of Machinery and Associated Technology", Barcelona, Spain.

Hammann, G.(1998) Modellierung des Abtragsverhaltens Elastischer Roboter-gefuehrter Schleifwerkzeuge. PhD thesis, University Stuttgart.

Iivarinen J; Visa, A. (1998) An Adaptive Texture and Shape Based Defect Classification. In the Proceedings of the 14th International Conference on Pattern Recognition, vol. 1, pp. 117-122, Brisbane, Australia

Lampinen, J; Smolander, S. (1994) Wood defect recognition with self-organizing feature selection. Intelligent Robots and Computer Vision XIII: Algorithms and Computer Vision, Proc. SPIE 2353, pp. 385-395.

Kneupner, K. (2002) Directcontrol. Ein Programmierkonzept fuer Roboterzellen. In Conference proceedings of Robotik, Ludwigs-burg, Germany.

Kneupner, K. (2004). Entwicklung eines Programmier und Steuerungskonzepts fuer Robotersysteme auf der Basis eines Umwel-tmodells. PhD thesis, Dortmund University. 
Koenig, W. (1996) Fertigungsverfahren Band 2 --- Schleifen, Honen, Laeppen. VDI Verlag. Krause, R. H. (2001) Monotone Multigrid Methods for Signorini's Problem with Friction. PhD thesis, Freie University Berlin.

Kreis, W; Kneupner, K. (2001a). Ein Simulationsgerechtes Prozessmodell fuer das Freiform Bandschleifen. Dortmund Germany.

Kreis, W; Kneupner, K. (2001b) Simulation des Bandschleifprozesses. Frontiers in Simulation, Simulationstechnik 15th. Symposium, pp 517-522,. Paderborn Germany.

Meyerhoff, M. (1998) NC-Programmierung fuer das kraftgesteuerte Bandschleifen von Freiform-flachen. PhD thesis, University Hannover.

Schueppstuhl, T. (2003) Beitrag zum Bandschleifen komplexer Freiformgeometrien mit dem Industrieroboter. PhD thesis, University Dortmund.

Suttmeier, F:T. (2001) Error Analysis for Finite Element Solutions of Variational Inequalities. PhD thesis, Dortmund University. 


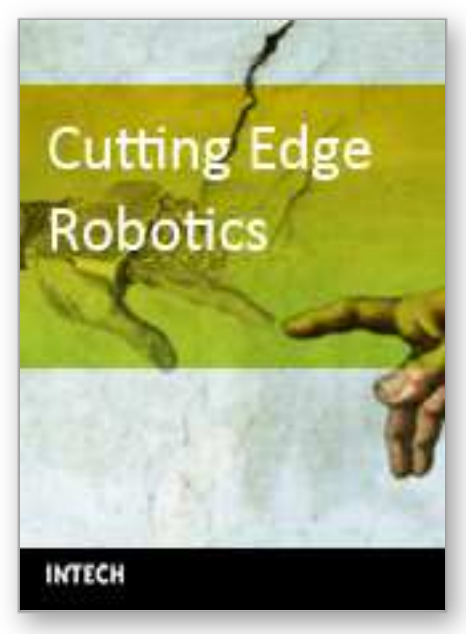

\section{Cutting Edge Robotics}

Edited by Vedran Kordic, Aleksandar Lazinica and Munir Merdan

ISBN 3-86611-038-3

Hard cover, 784 pages

Publisher Pro Literatur Verlag, Germany

Published online 01, July, 2005

Published in print edition July, 2005

This book is the result of inspirations and contributions from many researchers worldwide. It presents a collection of wide range research results of robotics scientific community. Various aspects of current research in robotics area are explored and discussed. The book begins with researches in robot modelling \& design, in which different approaches in kinematical, dynamical and other design issues of mobile robots are discussed. Second chapter deals with various sensor systems, but the major part of the chapter is devoted to robotic vision systems. Chapter III is devoted to robot navigation and presents different navigation architectures. The chapter IV is devoted to research on adaptive and learning systems in mobile robots area. The chapter V speaks about different application areas of multi-robot systems. Other emerging field is discussed in chapter VI - the human- robot interaction. Chapter VII gives a great tutorial on legged robot systems and one research overview on design of a humanoid robot. The different examples of service robots are showed in chapter VIII. Chapter IX is oriented to industrial robots, i.e. robot manipulators. Different mechatronic systems oriented on robotics are explored in the last chapter of the book.

\section{How to reference}

In order to correctly reference this scholarly work, feel free to copy and paste the following:

Bernd Kuhlenkoetter and Xiang Zhang (2005). A Robot System for High Quality Belt Grinding and Polishing Processes, Cutting Edge Robotics, Vedran Kordic, Aleksandar Lazinica and Munir Merdan (Ed.), ISBN: 386611-038-3, InTech, Available from:

http://www.intechopen.com/books/cutting_edge_robotics/a_robot_system_for_high_quality_belt_grinding_and _polishing_processes

\section{INTECH}

open science | open minds

\author{
InTech Europe \\ University Campus STeP Ri \\ Slavka Krautzeka 83/A \\ 51000 Rijeka, Croatia \\ Phone: +385 (51) 770447 \\ Fax: +385 (51) 686166 \\ www.intechopen.com
}

\author{
InTech China \\ Unit 405, Office Block, Hotel Equatorial Shanghai \\ No.65, Yan An Road (West), Shanghai, 200040, China \\ 中国上海市延安西路65号上海国际贵都大饭店办公楼 405 单元 \\ Phone: +86-21-62489820 \\ Fax: +86-21-62489821
}


(C) 2005 The Author(s). Licensee IntechOpen. This chapter is distributed under the terms of the Creative Commons Attribution-NonCommercial-ShareAlike-3.0 License, which permits use, distribution and reproduction for non-commercial purposes, provided the original is properly cited and derivative works building on this content are distributed under the same license. 